\title{
L’art écologique : une forme de médiation des sciences de la conservation?
}

\author{
Joanne Clavel
}

Écologie et art, Université de Liège, Faculté de philosophie et lettres, Département Arts et sciences de la communication, 4000 Liège, Belgique

Esthétique environnementale, art écologique, les expressions ne manquent pas pour circonscrire un champ de recherche relativement nouveau en France, mais en formation depuis longtemps outre-Atlantique. Il y est question d'esthétisation de l'espace public, ou bien de nouvelles formes de relations entre les arts, la recherche et l'éthique, ou bien encore de la place de l'esthétique dans les mobilisations associatives. Cet article s'inscrit plutôt dans le second courant en tentant un rapprochement entre la biologie de la conservation et les manifestations plurielles d'un art se réclamant de l'engagement écologique. NSS a traité cette question émergente à plusieurs reprises, notamment dans un éditorial intitulé « Artistes et scientifiques : soupçons et espoirs d'interdisciplinarité » (NSS, 16,3 [2008]), puis à travers d'autres publications, qu'il s'agisse d'études de cas, d'articles de synthèse ou de comptes rendus (NSS, 16, 2 [2008], 122-130; NSS, 16, 3 [2008], 279-281; NSS, 17, 3 [2009], 285-292). La tentative des auteurs de proposer une catégorisation des créations qui se revendiquent de $l^{\prime}$ « art écologique » trouvera-t-elle un écho dans le milieu artistique?

La Rédaction

Mots-clés :

recherche ;

biodiversité ;

médiation artistique ;

écologie ;

art écologique
Résumé - L'art, outil de médiation pour la science, est un nouveau fait auquel peu de recherches se sont intéressées. L'objectif de ce travail est de confronter deux courants issus du nouveau paradigme écologique que sont les sciences de la conservation et le mouvement d'art écologique. Si, d'un côté, l'écologie scientifique s'inscrit de plus en plus dans une démarche interdisciplinaire afin de concilier activités humaines et préservation de la nature, les créations des artistes écologiques synthétisent de leur côté les représentations, les pratiques sociales et les dimensions biologiques ou physiques d'un milieu. Il peut être utile de faire le point sur ces démarches.

\section{Keywords:}

research; biodiversity; artistic mediation; ecology; ecological art

\begin{abstract}
Ecological Art, a mediation agent in Conservation Sciences? Art as a means of scientific mediation is a new and unexplored phenomenon. Our ambition is to compare two trends deriving from the new ecological paradigm: Conservation Sciences and the Ecological Art Movement. While on the one hand scientific ecology increasingly turns to an interdisciplinary approach aiming to reconcile human activities and the preservation of nature, the ecological artists' creations on the other hand compound social representations, social practices and the environment. It may be useful to review these approaches and to understand how an artistic mediation of Ecology may provide a complementary approach to the classical popularization of science. We start by presenting the Ecological Art Movement, based on the latest major eco-art exhibition and illustrate our comments with artworks. We then describe the conservation tools available to scientists, given that conservation of global biodiversity depends on two major issues: the local preservation of biodiversity (through reservation/restoration) and the emergence of an ecological conscience among citizens (through reconciliation/reconnection). Third, we link ecoartworks to the " $4 \mathrm{R}$ " Conservation principles. And finally, we discuss the artistic mediation of Ecology and the potential emergence of ecological conscience through art.
\end{abstract}

Auteur correspondant : joanne.clavel@gmail.com 
La prise de conscience des menaces anthropiques pesant sur la biodiversité (Chapin et al., 2000) a entraîné dans les années 1980 la naissance d'une nouvelle discipline des sciences de l'écologie: la biologie de la conservation (Soulé, 1986). Devant l'urgence de la situation, les biologistes de cette discipline ont joué les « lanceurs d'alerte » auprès de la société et ont accompagné leurs discours scientifiques de valeurs (ibid.). Dans ce cadre, la vulgarisation de leurs travaux est le prolongement indispensable de leurs recherches. Il s'avère aujourd'hui que la vulgarisation scientifique classique ne procure pas les résultats escomptés (Simon, 2006), c'est-àdire une sensibilisation apte à modifier les comportements. Politiquement, des avancées notables sont à relever (Balmford et al., 2005). Mais elles ne s'accompagnent pas, à l'heure actuelle, d'une diminution de l'érosion de la biodiversité (Millennium Ecosystem Assessment, 2005). Certains auteurs expliquent cela par l'inefficacité de gestions imposées par le haut (top-down) [Fraser et al., 2006]. Mais l'une des raisons premières est que les objectifs identifiés par les écologues demandent une réorganisation sociétale sans précédent - notamment au niveau de l'économie de marché -, ce qui peut prendre du temps (Boullier, 2002 ; Latour, 2002)... De plus, la prise en main de cette question de société au niveau individuel est mitigée, peut-être parce que la communication en matière d'écologie s'accompagne le plus souvent d'un discours culpabilisant et moralisateur qui n'établit ni les conditions d'un dialogue ni, a fortiori, celles d'une médiation qui rende le public acteur (Caune, 1995). Dans cet article, nous tenterons d'étudier une nouvelle proposition de médiation par laquelle le public se sentirait plus concerné et donc peut-être plus à même de s'engager vers un changement de comportement ${ }^{1}$.

Dès les premières manifestations connues de l'art, l'homme s'inspire de la nature: les peintures murales néolithiques, par exemple, représentent des scènes de vie mêlant nature sauvage et domestiquée autour d'activités humaines. Or, il existe aujourd'hui un mouvement d'art contemporain qui s'inspire profondément de la nature tout en cherchant à délivrer un message de protection et de conservation de cette dernière. Il peut, en conséquence, être utile de faire le point sur ces deux démarches : « l'art écologique » d'un côté et, de l'autre, la biologie de la conservation. Dans un premier temps, nous présenterons le mouvement d'art écologique, son émergence et les différentes formes qu'il revêt actuellement. Dans un second temps, nous élargirons la biologie de la conservation aux sciences de la conservation et détaillerons ses quatre

1 Cet article a fait partiellement l'objet d'une communication lors de la journée d'étude : La Nature dans la ville : quels enjeux de communication? organisée par l'ISCC, Natureparif, le CNRS et l'Université Paris Sud en décembre 2008. Les recherches ont été menées dans le cadre d'une bourse postdoctorale à l'Université de Liège. outils majeurs (les « $4 \mathrm{R} »)$ : « réservation », restauration, réconciliation, reconnexion (Prévot-Julliard et al., 2011a ; Caplat et Coutts, 2011 ;Prévot-Julliard et al.,2011b).Enfin, nous analyserons les caractéristiques de l'art contemporain qui sont susceptibles de le rapprocher des objectifs des sciences de la conservation; cela nous amènera à revenir sur quelques exemples permettant de relier le cadre théorique des « $4 \mathrm{R}$ » à la démarche d'éco-artistes contemporains.

\section{L’art écologique}

\section{Présentation}

L'émergence d'un mouvement d'art contemporain dit écologique, à la fin du XX $X^{\mathrm{e}}$ siècle, est directement liée à la concentration des problèmes environnementaux contemporains : pollution, réchauffement climatique, extinction des espèces, etc. (Bower, 2007). L'art écologique prend historiquement ses sources aux États-Unis avec le mouvement précurseur du Earth Art à la fin des années 1960 (Ramade et Mangion, 2010). Partageant avec le Land Art des créations in situ qui proposent aux spectateurs une «immersion» dans la nature loin de l'institution muséale, il s'en distingue par un profond respect de l'environnement lors des créations artistiques. Si ce mouvement d'art écologique n'a jamais été défini avec clarté (Ramade, 2007), c'est qu'il demande à repenser les catégories institutionnelles (Margolin, 2005) afin de dépasser le simple thème de l'environnement (Kurt, 2004) et d'embrasser un point de vue plus large rassemblant objet, participation et action. Les artistes impliqués, par leur éthique écologique, leur prise de position dans l'espace public et les liens étroits qu'ils entretiennent avec les scientifiques ou les gestionnaires, cherchent à éveiller les consciences, voire à modifier le milieu de vie des hommes de façon durable (Margolin, 2005). C'est leur engagement à répondre aux enjeux sociétaux en conformité avec une conscience écologique qui structure actuellement ce courant. Bien qu'une dynamique similaire dans la communauté des artistes existe actuellement en Europe, au Japon et en Amérique latine (surtout au Brésil), l'art écologique n'y est pas encore réellement reconnu comme tel par le monde de l'art (commissaires d'expositions, critiques, marchands, universitaires $)^{2}$.

Dans la mesure où l'art écologique n'est pas le seul objet de cet article, il nous est impossible de présenter tous

\footnotetext{
2 Pour la France, évoquons la commissaire d'exposition Bénédicte Ramade (Acclimatation, Villa d'Arson, Nice, 2009, et L'Art de re-faire, fondation EDF, Paris, octobre 2010, février 2011), ainsi que le groupement associatif COAL (Coalition pour l'art et le développement durable): http://www.projetcoal.fr/ (consulté en août 2011).
} 
les artistes engagés dans ce mouvement (Spaid, 2002) ; il nous a donc semblé pertinent de l'aborder en prenant pour point de départ la dernière grande exposition dite d'art écologique : Badlands, reconnue comme telle par ses pairs. Cette exposition au Massachussetts Museum of Contemporary Art (MASS MoCa) a donné lieu à une publication ${ }^{3}$, rassemblant professionnels de l'art et scientifiques, qui avait pour objectif de mettre en évidence les caractéristiques et les tendances actuelles de ce mouvement. Nous reprendrons la typologie proposée par la commissaire de l'exposition, Denise Markonish, distinguant quatre tendances et nous illustrerons notre propos par la présentation de quelques artistes.

Commençons par les « historiens », qui décrivent un paysage et mettent leurs travaux en rapport avec son contexte et son histoire. Robert Adams, par exemple, photographie l'Amérique de l'Ouest en prenant à la lettre, pour guider son itinéraire, la métaphore de " The road less travelled (la route la moins empruntée). Il représente autant la beauté de la vie de tous les jours que les dévastations qu'opère l'homme sur le paysage. Il déconstruit la conception même de paysage, en photographiant des bâtiments, des routes, des déforestations, des déchets, etc. (Photo 1). Fabriquée par la peinture, la notion de paysage a en effet longtemps représenté une séparation entre la nature et les activités humaines, proposant la vision idéalisée d'une harmonie Homme-Nature. Les photographies de R. Adams s'inscrivent dans une "fin de la nature romantique et l'effacement des frontières entre les hommes et la nature » (Bright, 1992, p. 65).

La seconde catégorie est appelée par D. Markonish les « explorateurs », c'est-à-dire ces artistes qui vont à la rencontre de l'environnement, du paysage, et y examinent la place de l'homme. Que cela soit à une échelle macro ou microscopique, aux quatre coins du globe ou en bas de chez eux, ils cherchent à comprendre le monde qui les entoure, à l'explorer. Le cœur du projet de Jane D. Marsching, par exemple, pose la question : «Qu'estce que le pôle Nord ? » Elle a d'abord travaillé sur l'élaboration d'un site virtuel d'exploration du pôle Nord où les gens étaient invités à poser des questions et à échanger sur ce thème. Puis, en collaboration avec des scientifiques et des architectes, elle a imaginé ce que serait le pôle Nord dans cent ans, mélangeant ainsi les faits, les probabilités de réalisation et la pure fiction (Photo 2). Dans le contexte de réchauffement climatique actuel, qui touche plus fortement les hautes latitudes, l'Arctique subit de très rapides modifications. Dans Future North, J.D. Marsching imagine une possibilité d'avenir de ce milieu en se nourrissant des idées de l'écologie, du développement durable, ainsi que de données sociologiques et technologiques. Les hommes coloniseront-ils cet espace? Dans quelles conditions? Avec quelles nouvelles

\footnotetext{
3 Markonish (2008).
}

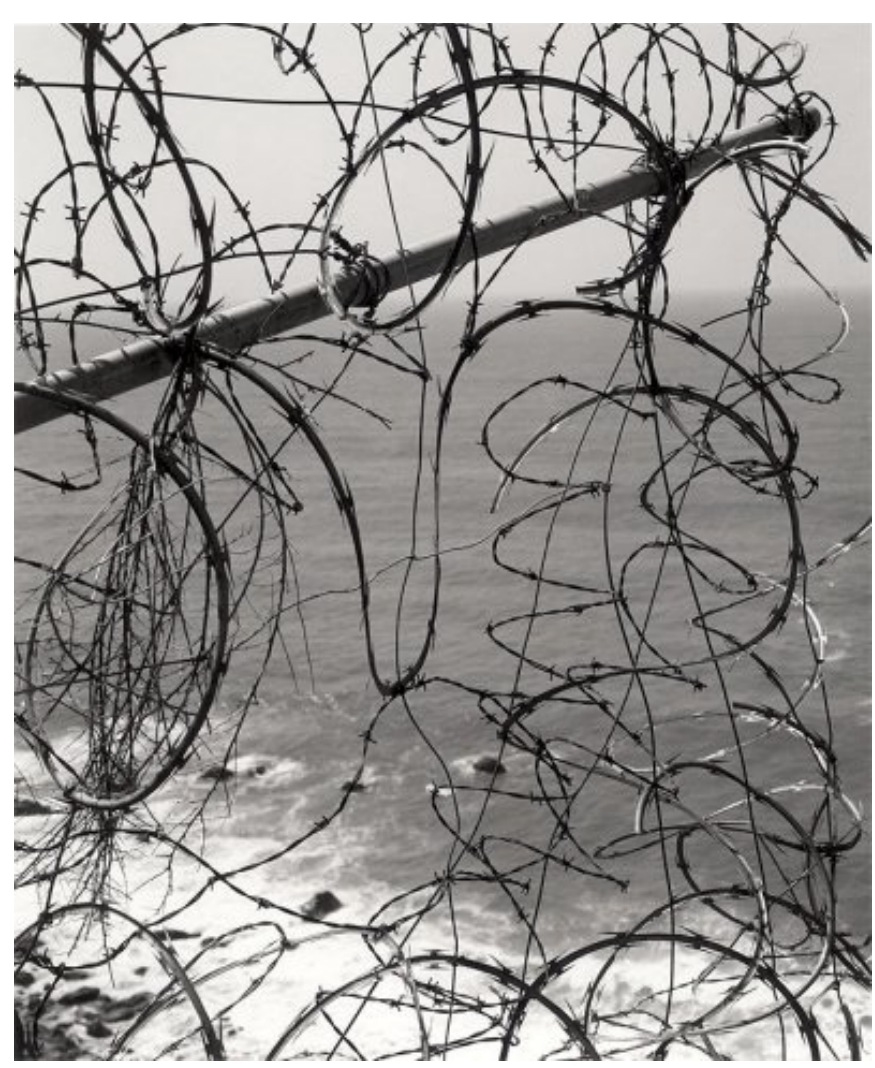

Photo 1. Robert Adams, Dead Plant Barbed Wire, 1983.

(C) Robert Adams. Courtesy Fraenkel Gallery, San Francisco and Matthew Marks Gallery, New York. Reproduction is strictly prohibited.)

technologies? L'océan Arctique deviendra-t-il une mer ouverte toute l'année?

Bien que tous les éco-artistes soient engagés dans la cause écologique, les « activistes » sont certainement les plus dynamiques du mouvement, car leurs œuvres portent directement sur la crise environnementale actuelle et son devenir. Ils alertent le public sur les problèmes environnementaux, cherchent parfois des solutions écologiques pour y remédier. De nombreux artistes opèrent par la représentation des désastres (fictifs ou réels) et posent ainsi la question : «Sommes-nous préparés ? - - c'est le cas dans les peintures théâtrales mêlant beauté et terreur de Leila Daw. Ils peuvent également chercher à informer sur des faits, comme J. Henry Fair. Ce dernier, à partir d'images vues du ciel, présente les dommages de l'industrie, tels que la pollution chimique des États-Unis (Photo 3). Pourtant, le spectateur est d'abord frappé par la beauté des images, avant de prendre conscience que ces couleurs rayonnantes proviennent de rejets ou de processus chimiques industriels polluant l'air, l'eau ou le sol pour des centaines d'années. C'est probablement dans l'écart entre ces deux valeurs - esthétique et écologique que réside la force de ces œuvres. 


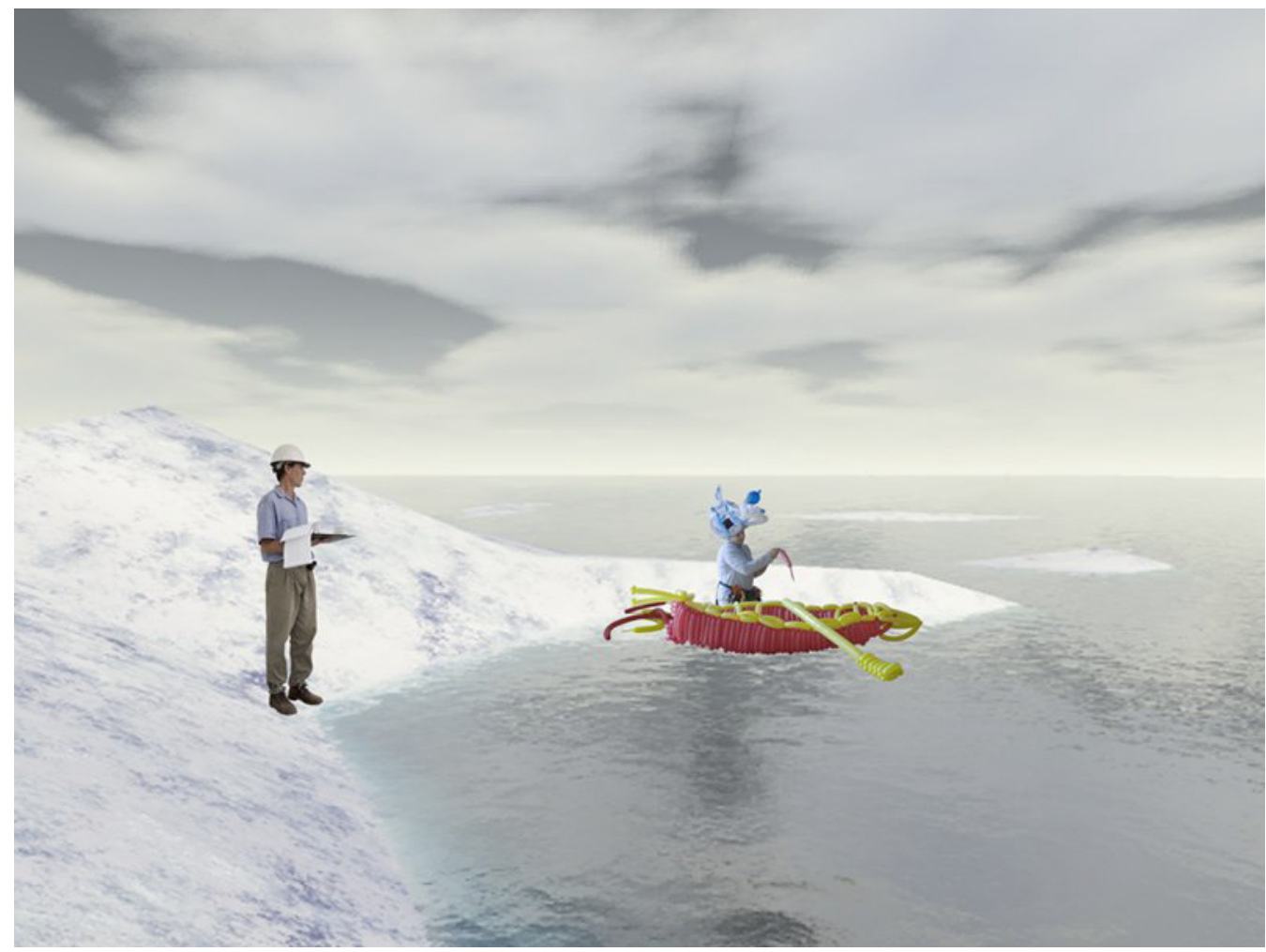

Photo 2. Jane D. Marsching, Arctic Then, 2006.

(C) Jane D. Marsching. Reproduction is strictly prohibited.)

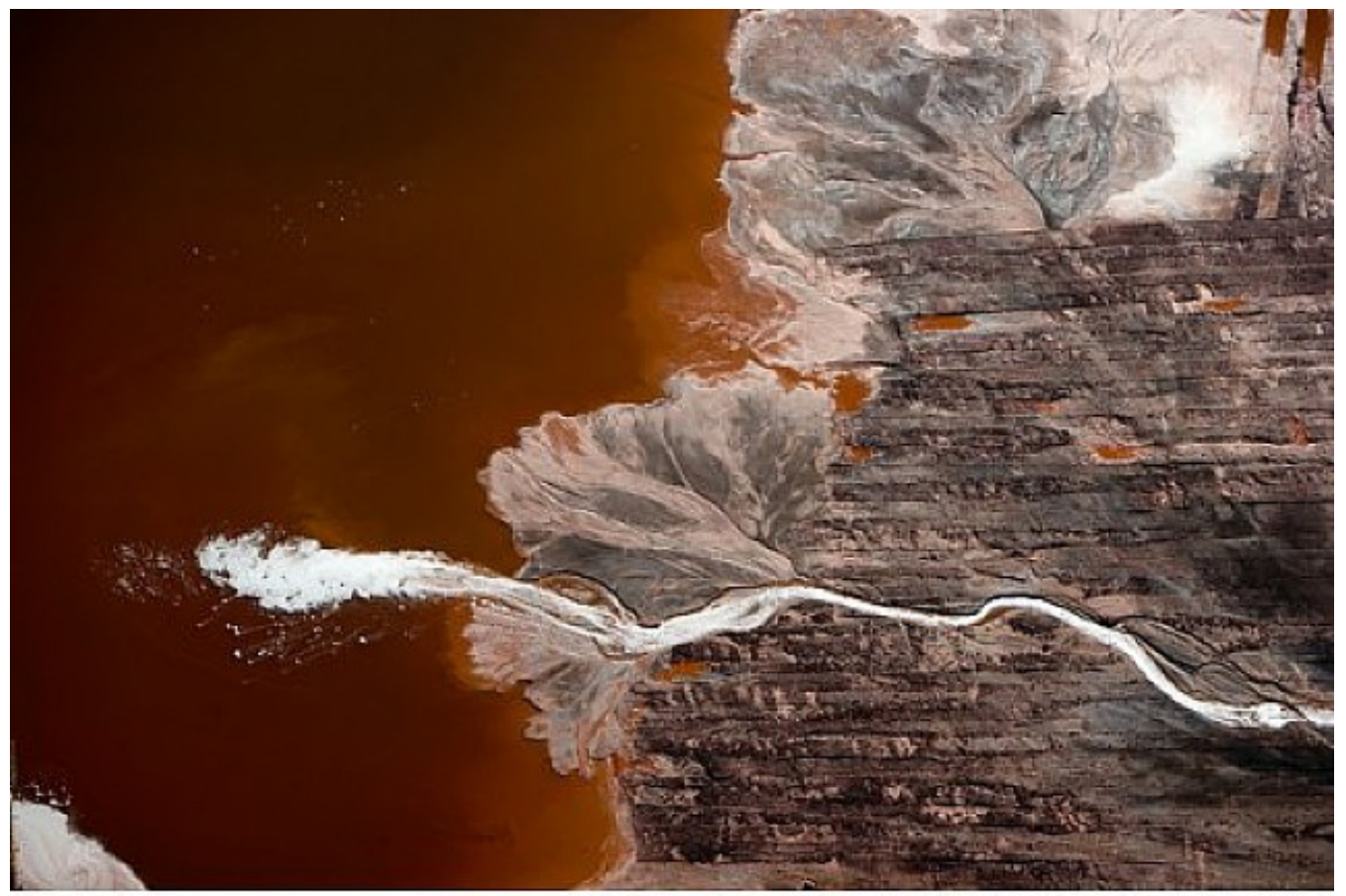

Photo 3. J. Henry Fair, Expectoration, 2005.

(C 2009 J. Henry Fair. Reproduction is strictly prohibited.) 


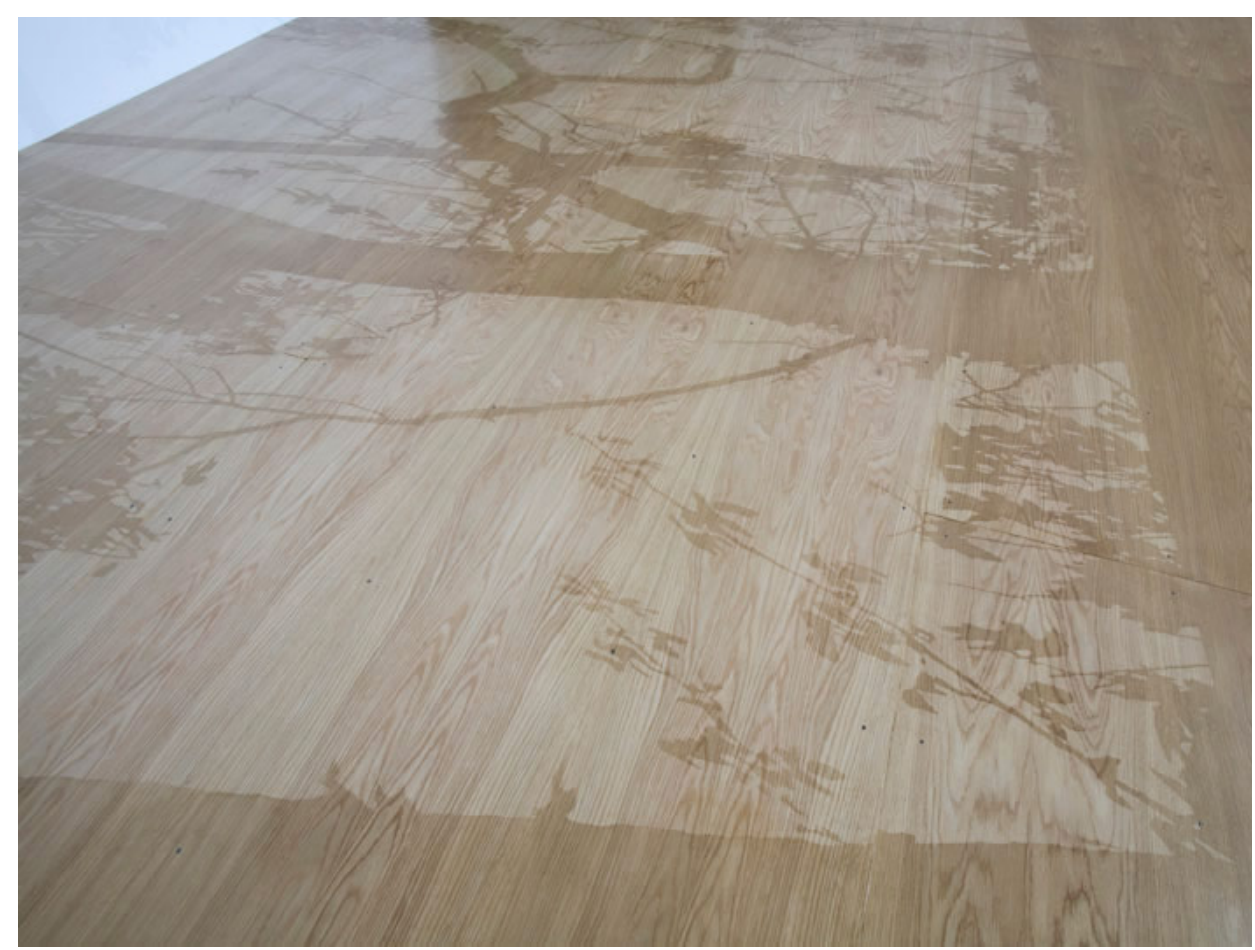

Photo 4. Mary Temple, Extended Afternoon, 2005.

(C Mary Temple. Courtesy of Mary Temple and Mixed Greens Gallery, New York. Reproduction is strictly prohibited.)

Enfin, les artistes dits «esthétiques » traitent de la beauté de la nature pour elle-même. Ils adhèrent à l'idée de merveilleux dans la nature et croient au pouvoir émotionnel de l'expérience de celle-ci. L'une d'entre eux, Mary Temple, joue sur la lumière. Elle recrée magnifiquement des ombres d'arbres en milieu intérieur et apporte directement la nature au musée. Mais le spectateur, contemplant doucement la découverte de ces ombres, réalise petit à petit qu'il n'y a pas de fenêtres, qu'elles sont simplement peintes... Ainsi, M. Temple fait aussi appel à la trace, à la mémoire, avec une résonance étrange et inquiétante en période de crise écologique, comme le souvenir d'un paradis perdu (Photo 4).

\section{Un art engagé}

On le voit, l'art écologique, tel qu'il se présente ici, se pose - et parfois de manière très explicite - comme le moyen d'une prise de conscience et d'un changement sociétal. Il s'inscrit à ce titre dans un débat qui le dépasse de beaucoup, et qui renvoie aux questions relatives à l'utilité de l'art, à la possibilité pour l'art de produire un changement chez le spectateur et, plus généralement, au rapport de l'art à la connaissance et à la vérité. L'art estil fin en soi ou moyen d'expression et de représentation? Il s'agit là d'une question importante pour notre propos, car elle touche à la possibilité même de «transmettre » quoi que ce soit par l'œuvre d'art, à la possibilité que l'art « soit» ou « fasse » médiation ${ }^{4}$.

L'art écologique s'inscrit très clairement dans une prise de position politique, par une éthique vis-à-vis de la nature basée sur un principe de responsabilité environnementale. Dans ce cadre, l'éthique environnementale apparaît comme un principe structurant l'expérience esthétique. L'art écologique s'identifie non pas par des pratiques $^{5}$, mais par son engagement écologique et sa finalité pragmatique : il a pour objectif de produire un effet sur les consciences, il est engagé vers le changement. Comme l'affirment Blanc et Lolive (2009, p. 289) :

«L'art environnemental peut se présenter comme une synthèse esthétique traduisant une volonté de "réparer" symboliquement l'environnement [...]. C'est là une façon d'intervenir sur la géographie, l'histoire, la biologie du site, bref : d'évoquer un nouveau rapport à celui-ci. [...]Il engage,

\footnotetext{
4 «La notion de médiation apparaît chaque fois qu'il y a besoin de décrire une action impliquant une transformation de la situation et du dispositif communicationnel, et non une simple interaction entre éléments, et encore moins une circulation d'un élément d'un pôle à un autre. [...] Avec ce recours, l'origine de l'action se déplace de l'actant destinateur ou des interactants vers un actant tiers: il y a communication par l'opération. » (Davallon, 2003, p. 44.)

5 Comme le Land Art, le Nouveau Réalisme ou, d'une manière générale, les courants du $X X^{\mathrm{e}}$ siècle.
} 
alors, non seulement l'expérience esthétique singulière et individuelle, mais aussi le jugement de goût politique et collectif. C'est en ce sens que l'espace public esthétique peut renouveler l'espace contemporain du politique. »

Il en résulte des œuvres adoptant des modes d'intervention et des pratiques très différents, dont le seul point commun serait l'engagement de l'artiste. Certaines œuvres développent un imaginaire fondé sur des jeux de représentations à partir de supports d'expression classiques tels que les tableaux. D'autres mettent l'accent sur les propriétés biologiques et écologiques d'une réalité, à partir d'œuvres éphémères ou en y intégrant des éléments de nature vivante. D'autres encore s'ouvrent complètement à l'ensemble du monde réel comme milieu d'expérience artistique. Elles intègrent ainsi une démarche participative ou inclusive du public qui se distingue de la relation frontale strictement contemplative et visuelle. Fel (2008, p. 281) considère que ce glissement de la contemplation à l'expérience caractérise "un nouveau paradigme $[. .$.$] , une expérience esthétique [...] qui porte$ sur des relations [...], sur des ensembles dynamiques délimités dans le temps, comme ce que nous donne à comprendre la description d'un écosystème ". L'idée de nature proposée est elle aussi multiple : nature refuge de la solitude, nature sauvage et vierge ou lieu à habiter, elle se trouve toujours liée plus ou moins fortement aux sciences environnementales.

Cette particularité du mouvement d'art écologique de se distinguer par son «message » et non par sa pratique pourrait représenter un atout majeur dans son rôle de médiateur de la conservation de la nature. En effet, l'art contemporain s'expose aujourd'hui à de nombreuses formes de rejet, et notamment parce qu'il a bafoué la normalité, « le sens commun de l'art », dont les critères sont principalement la beauté et la technique (Heinich, 1998). Or, si un même message est relayé par une multiplicité de formes artistiques, on peut s'attendre à ce qu'il soit entendu, ou du moins pressenti, par une multitude de publics.

Si les créations des artistes écologiques synthétisent une prise de conscience écologique, nous allons voir que, de son côté, afin de réguler l'empreinte de l'homme sur son environnement, la biologie de la conservation accueille maintenant dans ses laboratoires de nombreuses disciplines des sciences humaines (de l'éthique à la politique en passant par l'économie).

\section{Les outils de la conservation}

La biologie de la conservation est une discipline très particulière de l'écologie. Premièrement, elle traite des questions de maintien ou de restauration de la biodiversité ; c'est donc une discipline de gestion de crise où, en plus de l'étude des systèmes biologiques, des réponses en termes de méthodes de gestion sont attendues (Barbault, 1997). Ce point explique très certainement l'engagement des scientifiques de la conservation auprès des politiques dans l'élaboration de stratégies internationales de protection de la nature (ONU, Unesco...). Deuxièmement, elle s'appuie sur l'ensemble des sous-disciplines de l'écologie (génétique des populations, modélisation, biogéographie, écologie fonctionnelle, évolution...) et, depuis la fin du $X X^{\mathrm{e}}$ siècle, elle s'ouvre aux disciplines des sciences humaines en accueillant dans ses laboratoires sociologues, philosophes, économistes, etc. ; on parle alors de «sciences de l'environnement » ou de "sciences de la conservation ».

Les scientifiques peuvent avoir recours à quatre types d'actions (Prévot-Julliard et al., 2011a). Deux d'entre elles s'attachent directement à la biodiversité en utilisant, $\mathrm{d}^{\prime}$ une part, la mise en réserve ( "réservation ${ }^{6} »$ ) et, $\mathrm{d}^{\prime}$ autre part, la restauration de sites pollués, perturbés et déséquilibrés. Les deux autres types d'actions, plus récents, s'attaquent plus directement à la source du problème et cherchent à réduire rapidement les pressions anthropiques sur les milieux naturels par la réconciliation (Rosenzweig, 2003) et la reconnexion (Pyle, 2003). Nous allons détailler l'idée de nature et la place de l'homme que proposent ces « $4 R$ », afin de situer ensuite les différentes démarches artistiques dans ce cadre des outils scientifiques de la conservation de la nature.

\section{« Réservation »}

Le principe de mettre en réserve certains espaces a pour but de protéger la nature des actions négatives de $l^{\prime}$ homme et donc de conserver une "nature sauvage et vierge ", la célèbre wilderness en anglais (Delord, 2005). En français, le concept de "sauvage » dérive du latin sylvaticus, la forêt, ce qui est hors du monde humain. La nature est dominante dans cet espace et l'homme cherche à y intervenir le moins possible ; lorsqu'il intervient, c'est à son service, pour sa santé, tel un médecin bienveillant. Lors de la mise en réserve d'espaces, $c^{\prime}$ est d'abord le maintien de la richesse en espèces (avec un nombre d'espèces maximum) qui est recherché, mais ce sont surtout les espèces dites patrimoniales ou rares (avec une abondance maximale) qui seront privilégiées, d'où l'idée d'une « nature remarquable ». De plus, on préserve le fonctionnement des écosystèmes, c'est-à-dire les interrelations qui existent entre les espèces et leur milieu.

\footnotetext{
6 «Réservation » : anglicisme signifiant l'action de mettre en réserve des aires géographiques à protéger. Son utilisation en français porte à discussion; nous l'utilisons dans la lignée de l'HDR de Romain Julliard, Observatoires de biodiversité : un outil de recherche au cour des questions de sociétés, soutenue le 9 octobre 2007.
} 
La notion de « réservation » est avant tout politique, car cet outil n'a de sens que dans le cadre législatif. Dans un premier temps, les réserves naturelles étaient l'occasion d'une convergence d'intérêts scientifiques, éthiques, économiques et esthétiques. Or, d'une part, la logique du marché touristique provoque une fréquentation de plus en plus importante de ces réserves, inconciliable avec l'objectif écologique ; d'autre part, le principe même de " réservation » s'est trouvé remis en cause par les biologistes et ce, particulièrement dans le contexte des changements globaux, telles les perturbations climatiques (Rodrigues et al., 2004). Ainsi, la convergence d'intérêts à l'origine des réserves naturelles est à l'heure actuelle relative, et les réflexions sur les changements globaux ont impulsél'émergence de nouveaux outils sur de nouveaux territoires jusqu'alors exclus de tout procédé de conservation. On parle dès lors de la conservation de la « nature ordinaire » par opposition à la « nature remarquable » (Gaston et Fuller, 2007).

\section{Restauration}

Le principe de restauration ne s'attache pas à un espace particulier, comme c'est le cas pour la « réservation ». L'homme est au service de la nature, qu'elle soit « remarquable » ou « ordinaire ». Il emploie la science de l'écologie, à travers sa technicité et son ingénierie, au service des écosystèmes dans l'objectif de redonner à ces derniers un bon fonctionnement autonome. Comme le décrivent Palmer et al. (2004), il s'agit ici de réparer les écosystèmes endommagés et dégradés. Tandis que la « réservation » écartel'homme et ses dégradations potentielles par principe de prévention, la restauration agit là où des dommages ont été enregistrés afin de " guérir » les écosystèmes. La technique employée à cet usage est de l'ordre du « pilotage » de la nature, c'est-à-dire de l'infléchissement des processus naturels, ce que Larrère (2002) décrit comme « l'art du thérapeute qui aide l'organisme à guérir ». L'auteur précise que le pilotage, par définition, n'a jamais une efficacité absolue : il s'agit de « faire avec », et non de maîtriser. Le pilotage, qui pendant longtemps a été l'apanage du savoir-faire, de stratégies heuristiques, se retrouve maintenant ajusté par le développement des sciences et des connaissances, dans notre cas les sciences de l'écologie (ibid.).

\section{Réconciliation}

Le principe de réconciliation a pour objectif premier le développement durable des activités humaines (Rosenzweig, 2003). La réconciliation cherche à orienter les pratiques humaines de production de biens et de services vers un système symbiotique avec la nature. La priorité est cette fois-ci portée sur les activités humaines et non sur la nature. Pourtant, la conservation du fonctionnement des écosystèmes est essentielle au maintien des services écologiques rendus par la nature (régulation des eaux, du climat, etc.). Il ne s'agit pas de cibler les actions sur des espèces dites « particulières » ou « remarquables » (recherche d'abondance maximale), mais de maintenir le cortège d'espèces complémentaires nécessaires au fonctionnement viable des écosystèmes (recherche de communautés fonctionnelles, intégrant aussi la nature « ordinaire »). Ce principe concerne essentiellement les systèmes agraires occupant la majorité du territoire (Kareiva et al., 2007). Dans ces écosystèmes, la conservation appelle à une nouvelle gestion des ressources et de la production. Le concept de « pilotage » de la nature nous est une nouvelle fois utile ; il aura comme but, dans le cas présent, la production de biens et de services pour l'homme. Il s'oppose à un autre processus technique de production, celui de la « fabrication », majoritairement répandu dans les sociétés occidentales et qui, pour sa part, opère par artificialisation de la nature. En effet, la fabrication d'artifices est responsable des pollutions actuelles (air, eau, sol) lorsque ses effluents échappent au processus de fabrication (volontairement ou involontairement) [Larrère, 2002]. La réconciliation vise un changement des pratiques dans le cadre d'une nouvelle conception de l'intérêt public mêlant préoccupations écologiques et socioéconomiques. Elle s'inscrit donc dans une nouvelle idéologie politique.

\section{Reconnexion}

L'outil de la reconnexion se focalise sur la recherche d'un contact étroit et direct entre l'homme et la nature (Miller, 2006). En effet, maints auteurs soulignent qu'un nombre de plus en plus important de citoyens n'ont plus de contact avec la nature (Turner et al., 2004). Or, ce lien avec une forme de nature relève de la plus haute importance pour l'intérêt propre de l'homme, que ce soit pour sa santé (physique et psychique) ${ }^{7}$, sa qualité de vie ou pour les émotions qu'il lui procure. Si la restauration " rétablit » les écosystèmes endommagés, la reconnexion « rétablit » les citoyens dans une perception favorable de la nature qui, par la suite, guidera leurs engagements politiques. Afin de rétablir cette perception favorable et cette relation, la reconnexion utilise deux axes principaux : la vulgarisation de la science de l'écologie (éducation) et l'expérimentation de la nature par son contact direct. D'après Fel (2008), cependant, l'augmentation des connaissances via l'éducation joue un rôle prépondérant dans l'expérience de la nature : elle en accentue le goût ou le plaisir. Pour ce qui concerne la nature, dimension cognitive et dimension esthétique sont pour lui liées ; c'est ainsi qu'il parle $\mathrm{d}^{\prime}$ « esthétique verte ».

7 Pour le rôle de la nature dans la santé des hommes, voir Maller et al. (2006). 


\section{Les éco-artistes et les « $4 \mathrm{R}$ » de la conservation}

Sans être considéré intrinsèquement comme un élément du système d'éducation, l'art écologique a déjà contribué à améliorer la conciliation de l'homme avec son écosystème, notamment lors de la création des parcs nationaux ou dans le cadre de la restauration de sites.

Ce qui est en question ici est donc la possibilité pour l'œuvre d'art de produire un changement social à travers une fonction de communication ou de médiation; sa capacité, à travers une expérience (de création, de réception ou de « réception/création »), à produire une prise de conscience où l'homme (re)trouverait à la fois sa place et les critères du juste et du vrai. « Les artistes ont une position unique pour ressentir les changements environnementaux, ils peuvent synthétiser de nouvelles idées et faire la connexion entre différentes disciplines. Ils sont les pionniers d'une approche holistique ", écrit Maltisky (1992, p. 3). Enfin, est également en jeu la capacité de l'art à transmettre des informations et à faire œuvre de vulgarisation. Il faut alors penser l'œuvre à la fois comme forme de connaissance et comme espace de liberté propre à susciter une expérience.

Dans cette dernière partie, nous tenterons d'établir quelques-uns des liens qui, sous l'angle de l'approche esthétique ou de l'expérience de l'œuvre par le public, autorisent une première tentative pour comprendre comment l'art écologique peut jouer un rôle actif dans la conservation de la biodiversité.

\section{« Réservation »}

Dans l'histoire de la conservation, aucun artiste n'a, à ce jour, mieux articulé art et conservation qu'Ansel Adams (Turnage, 1980). A. Adams, photographe américain du début du $\mathrm{XX}$ e siècle, a produit de nombreux clichés de l'Amérique de l'Ouest. Ses travaux présentent la beauté d'une nature d'où toute trace humaine est écartée (wilderness). On voit bien ici que la notion culturelle de "nature » renvoie, au début du $X X^{\mathrm{e}}$ siècle, à un paysage d'une beauté "pure», sans altération humaine. Ses clichés du Yosemite Park ont grandement impulsé la création de parcs nationaux sur tout le territoire américain. A. Adams a été un activiste important dans plusieurs associations de conservation et sa notoriété a permis de faire pression sur les politiques afin de servir la conservation (ibid.). Avant lui, d'autres travaux avaient déjà eu une implication politique. Par exemple, les tableaux de paysages grandioses et inviolés de l'Ouest américain peints par Thomas Moran et Albert Bierstadt, ainsi que les photographies de la vallée du Yosemite de Carleton Watkins impressionnèrent le Congrès au point de peser sur la décision du président Abraham Lincoln de classer en parc naturel plusieurs sites exceptionnels dès 1864 (Ramade et Mangion, 2010).

Parallèlement, cet imaginaire de la nature vierge a influencé la biologie de la conservation dans ses premières mesures environnementales via la mise en réserve d'espaces afin de protéger la biodiversité. Comme le souligne Fel $(2008$, p. 310$)$, historiquement, « les lois instituant les parcs nationaux touchent des zones inhabitées, [...] non seulement pour des raisons pratiques, mais aussi parce que ces espaces sauvages sont fortement appréciés en raison de leur valeur de paysage "vierge" ». Donc, l'œuvre tout comme les démarches scientifiques et politiques participent à un imaginaire commun, l'artiste contribuant à renforcer cet imaginaire. L'expérience proposée par les photographies d'A. Adams relève d'un faceà-face avec la nature, de laquelle il faut se retirer. L'œuvre a ici clairement fonction de rassemblement. Elle sert les fins sociales en proposant une manière conventionnelle d'harmoniser les intérêts.

\section{Restauration}

La restauration de site a été maintes fois l'occasion de collaborations entre gestionnaires et artistes. Pionnière dans ce domaine, Patricia Johannson a réalisé de nombreux projets (Dallas, San Francisco, Corée...) mêlant beauté, écologie et mémoire collective. De leur côté, Commick travaille sur le problème de l'érosion des berges et Viet Ngo, sur le traitement des eaux usées (Bower, 2007). Le Pittsburgh Park Conservacy, pour sa part, a fait appel à des étudiants en art pour entreprendre des actions de restauration sur l'ensemble du site, composé de quatre grands parcs urbains de 1700 hectares dont 900 hectares de forêts naturelles. Leur collaboration visait à trouver des solutions créatives face aux problèmes environnementaux identifiés par les gestionnaires. Les principaux projets portaient sur le contrôle de l'érosion et la stabilisation des sols, ainsi que sur la recolonisation des plantes natives et l'élimination des exotiques. Dans cette expérience de restauration, l'implication des jeunes étudiants en art a mené à des solutions de gestion esthétiques et appréciées du public. Ici, c'est la beauté que l'artiste recherche, tout en considérant les contraintes imposées par la réhabilitation du milieu. Il participe à la beauté naturelle du site par l'artifice de son art: de ce point de vue, son intervention est comparable à celle d'A. Adams. Elle s'en distingue néanmoins par le fait que l'artiste produit le paysage plutôt qu'il ne le saisit, et ce, de manière concrète. De plus, ces jeunes citadins se sont imprégnés de la forêt, établissant un contact, une relation particulière avec la nature qui s'est prolongée dans le temps (au-delà de leur formation artistique) par de fréquentes visites en famille ou entre amis dans un but principalement récréatif. Les attachements vis-à-vis de 
ces lieux sont d'autant plus importants qu'ils sont partagés par le cercle personnel, familial et amical.

\section{Réconciliation}

Réconcilier les activités humaines et la biodiversité sur la majeure partie du territoire est très certainement l'un des enjeux les plus complexes des sciences de la conservation, en raison de l'immense inertie du système économique et social moderne. C'est dans ce cadre que Prue Lang, une chorégraphe australienne, engage depuis 2008 le processus de création Sustainable Dance Performance.

«Avec ce projet chorégraphique - explique-t-elle-, j'amène les questions et défis du développement durable à la scène. Je me suis donné comme objectif de rendre ce moment dansé autonome en termes d'énergie : les corps des danseurs et leurs mouvements deviendraient sources d'énergie pour les besoins en électricité de la pièce (éclairage, sonorisation, etc. $)^{8}$. "

La relation au corps est modifiée : objet plastique du travail chorégraphique, il devient aussi source d'énergie projetant, par un savant mélange de technologie et d'écologie, un théâtre pour demain. À côté des réponses artistiques aux problèmes énergétiques, nombreux sont les artistes qui se sont emparés de la question des déchets et du recyclage. Si l'idée de récupération et la dénonciation de notre civilisation de (sur)consommation était déjà répandue avec des mouvements comme l'Art brut, l'Arte Povera ou le Pop Art, les éco-artistes explorent des voies qui vont de la performance (nettoyage des rives d'un fleuve) au cabinet de curiosités composé de détritus chez Mark Dion, du recyclage des déchets en les revalorisant comme matière première de la créativité artistique pour La Réserve des $\operatorname{arts}^{9}$ à la mise en valeur de processus de décomposition chez Gérard Titus-Carmel.

\section{Reconnexion}

Enfin, la reconnexion de l'homme avec la nature peut s'effectuer par son contact direct. L'expérience de la nature touche profondément, par l'ensemble des sens. L'émotion ainsi libérée par la nature élargirait le champ affectif de la personne «troublée » (Foisy, 2008). Cependant, pour la plupart des citadins, le déplacement physique n'est pas rendu facile et, en milieu urbain, la nature n'est pas reconnue comme telle. De plus, un contact rompu depuis de nombreuses années peut entraîner une certaine peur de la nature sauvage ou une stigmatisation de certaines catégories de la biodiversité, par exemple les arthropodes. Les éco-artistes peuvent alors emmener leur vision de la nature au cœur des villes et servir de médiateurs de la cause écologique. Leur champ d'action peut aller de la présentation traditionnelle de

8 Observatoire des nouveaux médias (2010).

9 Cf. http://lareservedesarts.org leurs œuvres, en galeries ou en musées, à l'exposition en plein air de leurs travaux ou à une intervention physique avec le public, par des happenings ou des "sculptures sociales », pour reprendre l'expression de Joseph Beuys. En 1982, à la Documenta de Kassel, J. Beuys invitait le public à replanter 7000 chênes dans la ville et ses alentours. Chaque chêne était associé à une colonne de basalte dans un parc de Kassel ; les colonnes étaient disposées en tas au début de l'action, puis plantées chacune au pied d'un arbre au cours du déroulement de la sculpture sociale. Cette initiative s'est poursuivie pendant des années et a inspiré d'autres campagnes de reforestation impliquant le public. On note, ici, la participation du public à l'œuvre et à l'acte écologique, lesquels éveillent ses sens (vision, toucher, odorat, kinesthésie) et permettent peut-être une prise de conscience plus durable.

L'utilisation du corps comme source créatrice a laissé le monde de la danse relativement en dehors des débats sur la dichotomie entre nature et culture. La danse semble particulièrement pertinente dans le mouvement d'écoartistes pour repenser notre lien à la nature, notre ouverture à l'altérité le temps d'une expérience. Il existe une grande diversité d'approches, mais nous pouvons évoquer ici le travail pionnier d'Anna Halprin et celui de Jennifer Monson. A. Halprin focalise son travail sur le ressenti, avec des techniques corporelles qui convoquent le corps à une " écoute » du milieu dans lequel elle évolue, in situ, au contact direct avec la nature, et ce, grâce à son plateau de danse au milieu des arbres ou lors de ses créations en forêt, sur la plage ou dans un parc urbain (Caux et Raspail, 2006). J. Monson, chorégraphe, travaille aussi avec de nombreuses techniques d'improvisation et in situ, elle nourrit fortement sa création de connaissances scientifiques et en particulier biologiques (Monson, 2006). En 2005, à New York, elle crée iLAND ${ }^{10}$, un laboratoire de rencontre entre danse et écologie qui accueille chaque année de nouveaux artistes pour sensibiliser le public urbain.

Les quelques exemples développés ici montrent que les relations entre art et écologie sont d'ores et déjà nouées de diverses manières : les éco-artistes ont souvent sollicité scientifiques et gestionnaires pour nourrir leur création. De leur côté, les sciences de la conservation sont demandeuses de nouveaux moyens de communication auprès des citoyens afin d'envisager une conscientisation des problèmes écologiques qui mènerait à des changements comportementaux. Dans la mesure où l'œuvre d'art est porteuse de possibilités de communication que $d^{\prime}$ autres formes de médiation ne proposent pas, on peut estimer que la médiation artistique des sciences de la conservation est prometteuse, et ce, à travers deux axes pertinents selon les pratiques artistiques.

\footnotetext{
10 Interdisciplinary Laboratory for Art Nature and Dance : http:/ / www.ilandart.org/ (consulté en août 2011).
} 
Dans un premier axe, la conservation s'oriente vers une médiation artistique pour des raisons pédagogiques. Le regard que porte le spectateur sur la nature a été sensiblement modifié par les canons ou modèles de l'histoire du paysage pictural (Grimaldi, 1982), à tel point que l'on peut estimer que la peinture a modifié nos goûts et nos attentes par rapport au paysage. Par ailleurs, certains écoartistes, en s'inspirant des sciences de l'écologie dans leur activité de création, contribuent directement à la transmission des idées, des interrogations et des savoirs issus des sciences écologiques. L'imagination et la poésie deviennent des relais de la culture écologique. Or, cette approche cognitive augmente l'appréciation et le plaisir que peut dégager l'œuvre à l'instant de sa visualisation, et elle éveille de surcroît la curiosité ou l'envie du récepteur d'entrer en contact direct avec la nature. L'expérience signifiante $^{11}$ se joint aux expériences sensibles et esthétiques. Précisons que cette augmentation de connaissance par l'écologie peut se faire selon deux voies : celle des sciences naturelles; celle des sciences de l'écologie et de l'évolution.

Selon un second axe, l'art écologique permet l'expérience esthétique de la nature, même en milieu urbain ${ }^{12}$, où celle-ci est présente sous une forme majoritairement jardinée et domestiquée, mais dont le citadin attentif à son environnement peut encore en observer les traces éparses de spontanéité. La pratique de l'art écologique sous forme d'expérience permet l'inclusion du récepteur dans l'œuvre en sollicitant l'ensemble de ses facultés : sensorielles, émotionnelles, psychologiques et cognitives. Or, l'esthétique de « présentation » de la nature est un plaisir parfois qualifié de supérieur à celui de la seule représentation, puisqu'il est associé à " une expérience sensorielle, dans une relation libre, désintéressée et indépendante de toute représentation » $(\mathrm{Fel}, 2008$, p. 344).

$\mathrm{Si}$, selon ces deux axes, l'art écologique participe à la diffusion des notions écologiques et aux changements de perception du public, il pourrait peut-être, par son pouvoir émotionnel fort, provoquer des changements de comportement.

\section{Remerciements}

Nous remercions chaleureusement Christine Servais, notre tutrice pendant nos deux années postdoctorales à

\footnotetext{
11 Voir les travaux de John Dewey sur l'émotion qui naîtrait à la fois de l'expérience de la contemplation esthétique et de l'expérience signifiante. «C'est dans l'art, comme mode d'activité dont la signification est susceptible d'être immédiatement appréciée, que réside le parfait accomplissement de la nature ; la science est la servante qui conduit les événements naturels à cette heureuse issue. » (Dewey, 2010, p. 58.)

12 Telle est la démarche de l'artiste lyonnaise Céline Dodelin.
}

l'Université de Liège, ainsi que les artistes qui nous ont autorisée à illustrer cet article d'une de leurs œuvres, à savoir Robert Adams, Jane D. Marsching, J. Henry Fair et Mary Temple.

\section{Références}

Balmford, A., Bennun, L., Brink, B.T., Cooper, D., Côté, I.M., Crane, P., Dobson, A., Dudley, N., Dutton, I., Green, R.E., Gregory, R.D., Harrison, J., Kennedy, E.T., Kremen, C., Leader-Williams, N., Lovejoy, T.E., Mace, G., May, R., Mayaux, P., Morling, P., Phillips, J., Redford, K., Ricketts, T.H., Rodríguez, J.P., Sanjayan, M., Schei, P.J., van Jaarsveld, A.S., Walther, B.A., 2005. The convention on biological diversity's 2010 target, Science, 307, 5707, 212-213.

Barbault, R., 1997. Biodiversité : introduction à la biologie de la conservation, Paris, Hachette.

Blanc, N., Lolive, J., 2009. Vers une esthétique environnementale : le tournant pragmatiste, Natures Sciences Sociétés, 17, 3, 285-292.

Boullier, D., 2002. Le projet cosmopolitique, in Collin, J.-F. (Ed.), La Nature n'est plus ce qu'elle était, Paris, Cosmopolitiques / La Tour-d'Aigues, L'Aube, 7-9.

Bower, S., 2007. Greenmuseum, musée d'art environnemental en ligne, in Lolive, J., Blanc, N. (Eds), Esthétique et espace public, Paris, Cosmopolitiques / Apogée, 17-28.

Bright, D., 1992. The machine in the garden revisited: American environmentalism and photographic aesthetics, Art Journal, 51, 60-71.

Caplat, P., Coutts, S., 2011. Integrating ecological knowledge, public perception and urgency of action into invasive species management, Environmental Management, 48, 5, 878881.

Caune, J., 1995. Culture et communication: convergences théoriques et lieux de médiation, Grenoble, PUG.

Caux, J., Raspail, T., 2006. Anna Halprin à l'origine de la performance, Paris, Panama.

Chapin, F.S., Zavaleta, E.S., Eviner, V. T., Naylor, R. L., Vitousek, P.M., Reynolds, H.L., Hooper, D.U., Lavorel, S., Sala, O.E., Hobbie, S.E, Mack, M.C., Diaz, S., 2000. Consequences of changing biodiversity, Nature, 405, 234-242.

Davallon, J., 2003. La médiation: la communication en procès ?, in Thonon, M. (Ed.), Médiation et médiateurs, Médiation et information, 19, 37-59.

Delord, J., 2005. La "sauvageté»: un principe de réconciliation entre l'homme et la biosphère, Natures Sciences Sociétés, 13, 3, 316-320.

Dewey, J., 2010 L'Art comme expérience, Paris, Gallimard.

Fel, L., 2008. L'Esthétique verte: de la représentation à la présentation de la nature. Thèse de doctorat en philosophie, Université Paris 1 Sorbonne, Paris. Publiée en 2009 aux éditions Champ Vallon (Seyssel).

Foisy, S., 2008. Paysage à la croisée de l'expérience artistique et de l'expérience esthétique, in Erzen, J.N. (Ed.), Proceeding of the XVIIth International Congress of Aesthetics, "Aesthetics Bridging Cultures », 9-13 July 2007, Ankara, SANART, t. 1, $1-12$. 
Fraser, E.D.G, Dougill, A.J., Mabee, W.E., Reed, M., McAlpine, P., 2006. Bottom up and top down: Analysis of participatory processes for sustainability indicator identification as a pathway to community empowerment and sustainable environmental management, Journal of Environmental Management, 78, 114-127.

Gaston, K.J., Fuller, R., 2007. Biodiversity and extinction: Losing the common and the widespread, Progress in Physical Geography, 31, 213-225.

Grimaldi, N., 1982. L'esthétique de la belle nature, in Dagognet, F. (Ed.), Mort du paysage ? Philosophie et esthétique du paysage, Seyssel, Champ Vallon.

Heinich, N., 1998. Le Triple Jeu de l'art contemporain, Paris, Éditions de Minuit.

Kareiva, P., Watts, S., McDonald, R., Boucher, T., 2007. Domesticated nature: Shaping landscapes and ecosystems for human welfare, Science, 316, 5833, 1866-1869.

Kurt, H., 2004. Aesthetics of sustainability, in Strelow, H. (Ed.), Ecological Aesthetics, Art in Environmental Design: Theory and Practice, Basel, Birkhauser, 238-241.

Larrère, R., 2002. Agriculture : artificialisation ou manipulation de la nature?, in Collin, J.-F. (Ed.), La Nature n'est plus ce qu'elle était, Paris, Cosmopolitiques / La Tour-d'Aigues, L'Aube, 158-174.

Latour, B., 2002. Cosmopolitique, quels chantiers?, in Collin, J.-F. (Ed.), La Nature n'est plus ce qu'elle était, Paris, Cosmopolitiques / La Tour-d'Aigues, L'Aube, 15-26.

Maller, C., Townsend, M., Pryor, A., Brown, P., St Leger, L., 2006. Healthy nature healthy people: "Contact with nature" as an upstream health promotion intervention for populations, Health Promotion International, 21, 1, 45-54.

Maltisky, B., 1992. Fragile Ecologies: Contemporary Artists' Interpretations and Solutions, New York, Rizzoli.

Margolin, V., 2005. Reflections on art and sustainability, in Smith, S. (Ed.), Beyond the Green: Toward a Sustainable Art, Chicago, Smart Museum of Art, University of Chicago / New York, Independent Curators International, 20-29.

Markonish, D., 2008. Badlands: New Horizon in Landscape, North Adams, MASS MoCA.

Millennium Ecosystem Assessment, 2005. Ecosystems and Human Well-being: Synthesis, Washington (DC), Island Press (http:/ / www.millenniumassessment.org/en/index.html [Synthesis Reports; Overall synthesis], consulté en octobre 2010).

Miller, J.R., 2006. Restoration, reconciliation, and reconnecting with nature nearby, Biological Conservation, 127, 356-365.

Monson, J., 2006. Bird Brain Dance : entretien avec Jennifer Monson par N. Galeota-Wozny, Scientifiquement danse, Nouvelles de danse, 53, 212-236.

Observatoire des nouveaux médias, 2010. Conférence de Prue Lang, ENSAD et Université de Paris VIII : http://www.arpla.fr/odnm/?page_id=6038 (consulté en août 2011).

Palmer, M., Bernhardt, E., Chornesky, E., Collins, S., Dobson, A., Duke, C., Gold, B., Jacobson, R., Kingsland, S., Kranz, R., Mappin, M., Martinez, M.L., Micheli, F., Morse, J., Pace, M., Pascual, M., Palumbi, S., Reichman, O.J., Simons, A., Townsend, A., Turner, M., 2004. Ecology for a crowded planet, Science, 304, 5675, 1251-1252.

Prévot-Julliard, A-C., Clavel, J., Teillac-Deschamps, P., Julliard, R., 2011a. The need for flexibility in conservation practices: Exotic species as an example, Environmental Management, $47,3,315-321$.

Prévot-Julliard, A-C., Clavel, J., Teillac-Deschamps, P., Julliard, R., 2011b. Exotic species, experienced and idealized nature, Environmental Management, 48, 5, 882-884.

Pyle, R.M., 2003. Nature matrix: Reconnecting people with nature, Oryx, 37, 206-214.

Ramade, B., 2007. Mutation écologique de l'Art ?, in Lolive, J., Blanc, N. (Eds), Esthétique et espace public, Paris, Cosmopolitiques / Apogée, 31-42.

Ramade, B., Mangion, É., 2010. Acclimatation: Green Pandemonium, Blou, Monografik / Nice, Villa Arson.

Rodrigues, A.S.L., Andelman, S.J., Bakarr, M., Boltani, L., Brooks, T., Cowling, R., Fishpool, L.D.C., da Fonseca, G.A.B., Gaston, K., Hoffman, M., Long, J.S., Marquet, P.A, Pilgrim, J.D., Pressey, R.L., Schipper, J., Sechrest, W., Stuart, S.N., Underhill, L.G., Waller, R.W., Watts, M.E.J., Yan, X., 2004. Effectiveness of the global protected area network in representing species diversity, Nature, 428, 640643.

Rosenzweig, M.L., 2003. Reconciliation ecology and the future of species diversity, Oryx, 37, 194-205.

Simon, S., 2006. Systemic educational approaches to environmental issues: The contribution of Ecological Art, Systemic Practice and Action Research, 19, 143-157.

Soulé, M., 1986. Conservation Biology: The Science of Scarcity and Diversity, Sunderland, Sinauer Associates.

Spaid, S., 2002. Ecovention: Current Art to Transform Ecology, [s.1.], Greenmuseum.org / Cincinnati $(\mathrm{OH})$, Contemporary Arts Center / [S.1.], Ecoartspace (http://greenmuseum.org/c/ ecovention, consulté en août 2011).

Turnage, R., 1980. Ansel Adams: The Role of the Artist in the Environmental Movement. Reprinted courtesy of the Wilderness Society from The Living Wilderness: http:// www.anseladams.com/ansel-adams-the-role-of-the-artistin-the-environmental-movement/

Turner, W.R., Nakamura, T., Dinetti, M., 2004. Global urbanization and the separation of humans from nature, Bioscience, 54, 585-590.

Reçu le 19 novembre 2009. Accepté le 6 octobre 2011. 